ARTÍCULO ESPECIAL

\title{
Leches infantiles en España durante los años ochenta: impacto de las recomendaciones internacionales
}

\section{Josep Boatella* y Ricard Bou}

Departament de Nutrició i Bromatologia, Facultat de Farmàcia, Universitat de Barcelona, Barcelona, España

Recibido el 23 de noviembre de 2011; aceptado el 9 de mayo de 2012

\section{PALABRAS CLAVE} Dietética infantil; Leches infantiles; Leches en polvo; Composición;

Historia

\section{KEYWORDS}

Infant dietetics; Infant milk; Powdered milk; Composition; History

\begin{abstract}
Resumen
Se analiza la información existente sobre las leches infantiles (adaptadas, de seguimiento y especiales) comercializadas en España durante los años ochenta. Sobre un total de 74 muestras, se han aplicado análisis estadísticos con base en los datos de composición (proteínas, grasas, hidratos de carbono, vitaminas y minerales) y valor calórico, así como el grado de innovación de los productos (por modificación de fórmulas existentes $\mathrm{y} / \mathrm{o}$ adición de nuevos ingredientes). Los resultados obtenidos muestran un proceso de mejora en la diferenciación de las leches adaptadas y de seguimiento y en la adecuación a las recomendaciones internacionales publicadas.

(c) 2011 Asociación Española de Dietistas-Nutricionistas. Publicado por Elsevier España, S.L. Todos los derechos reservados.
\end{abstract}

Infant Milk Available in Spain in the 1980's: Impact of International Recommendations

\begin{abstract}
The information available on infant milk (adapted, follow-on and special) marketed in Spain during the 1980's has been analyzed. Statistical analyses have been carried out, taking into account the composition data of 74 formulas (protein, fat, carbohydrates, vitamins and minerals), and energy value, as well as the degree of innovation of the product (by modification of the existing formulas and /or by the addition of new ingredients). The results showed the process of a better differentiation between adapted and follow-on milks with time, and the adaptation to the published international recommendations.

(c) 2011 Asociación Española de Dietistas-Nutricionistas. Published by Elsevier España, S.L. All rights reserved.
\end{abstract}

\footnotetext{
*Autor para correspondencia.

Correo electrónico: boatella@ub.edu (J. Boatella).
} 


\section{Introducción}

Como continuación de anteriores trabajos ${ }^{1-3}$, en este artículo se describen las características y los tipos de leches infantiles comercializadas en España durante la década de los ochenta, a la vez que se incluye la relación de las principales empresas elaboradoras. Cabe destacar que durante ese periodo se inició una nueva etapa de la alimentación infantil, gracias a la publicación de diversas recomendaciones de carácter internacional que contribuyeron muy significativamente a la tipificación y la mejora higiénico-nutricional de los preparados destinados a la sustitución de la leche materna.

Ante los progresos de la pediatría y de las ciencias de la nutrición, y también porque hasta entonces se carecía de criterios de normalización (con la excepción de algunos documentos previos, como los de la Food and Drug Administration [FDA] de 1967 y la Sociedad Alemana de Pediatras de 1973), dos organismos de carácter internacional afrontaron el problema de la calidad y la idoneidad de las leches destinadas a la alimentación infantil. En este sentido, la XXXIV Asamblea de la OMS aprobó en 1981 un "Código Internacional de comercialización de sucedáneos de la leche materna" con el objetivo de "contribuir a proporcionar a los lactantes una nutrición segura y suficiente, promoviendo la lactancia natural y asegurando el uso correcto de los sucedáneos de la leche materna”. Por su parte, unos años antes, la European Society for Pediatric Gastroenterology, Hepatology and Nutrition (ESPGHAN), creada en 1968, había encomendado a un grupo de expertos la redacción de un informe sobre las leches adaptadas y la alimentación complementaria, que se publicó en 1977,1981 y $1982^{4-6}$. En esos documentos se definían los diferentes tipos de leches (formulaciones starting y follow-up), se recomendaba la eliminación de los términos "maternizadas" y "humanizadas" y su sustitución por la expresión "leches adaptadas", se señalaban unos valores de densidad calórica (proteínas y lípidos) y se indicaba también que: a) las fórmulas deben ser isotónicas; b) los hidratos de carbono utilizados han de ser disacáridos y oligosacáridos (para aportar energía sin aumentar la osmolaridad), de modo que se excluía el uso de almidón; c) no se debe utilizar miel ni los denominados "factores de crecimiento"; d) las leches no deben acidificarse, y e) la grasa puede contribuir al aporte energético en hasta un $50 \%$. A partir de 4-6 meses se recomendaba la introducción de una alimentación mixta de la que formaban parte las denominadas leches de seguimiento o continuación. Para ambos tipos de leche, la ESPGHAN recomendó, además, unos contenidos en ácido linoleico, minerales y vitaminas.

En relación con la formulación de productos, algunas de las cuestiones que entonces despertaban mayor interés eran, básicamente, aspectos relacionados con la osmolaridad y la carga renal, el contenido en caseína y el perfil en aminoácidos, la adición de aceites ricos en ácidos poliinsaturados, el enriquecimiento en vitaminas (D, A, E, K, C, fólico, $\mathrm{B}_{6}$ ), minerales y elementos traza y la idoneidad de la adición de carnitina, colina, inositol y nucleótidos ${ }^{7}$.

Por otra parte, cabe destacar que en 1976 se había aprobado en nuestro país la "Reglamentación técnico-sanitaria para la elaboración, circulación y comercio de los prepara- dos alimenticios para regímenes dietéticos y/o especiales"8, que incluía estos productos dentro del grupo de alimentos que satisfacen las exigencias fisiológicas especiales de nutrición de las personas sanas (lactantes y poslactantes) y definía las leches humanizadas (o maternizadas). La aprobación de ese decreto (modificado en diversas ocasiones durante la década de los ochenta), habría de tener importantes consecuencias para este sector, puesto que, entre otros aspectos, abrió las puertas a nuevos canales de distribución y venta de estos productos independientemente de las oficinas de farmacia.

\section{Empresas elaboradoras}

Las principales empresas elaboradoras y/o distribuidoras de preparados destinados a la población infantil en nuestro país durante el periodo eran: Alter S.A., Cheminter, Edda, FAES S.A., José Sánchez Peñate, Mead Johnson (Bristol Myers S.A.E.), Nestlé A.E.P.A., Nogalda S.A. (Milupa), Nutricia Ibérica S.A., Ordesa S.A., Ulta, UNIASA S.A. y Wander S.A.E. Algunas iniciaron su actividad en este sector (Alter, Uniasa, José Sánchez, Edda) o eran distribuidoras de marcas extranjeras (Nutricia Ibérica, Mead Johnson), mientras que otras cesaron en ella durante ese periodo (Ulta, FAES). En este trabajo se omite el comentario de algunas de ellas por haberlas tratado ya en un artículo anterior ${ }^{2}$.

\section{Alter S.A.}

Laboratorios fundados en Madrid en 1939 por el farmacéutico riojano J.J. Alonso Grijalba e inicialmente dedicados a la elaboración de preparados vitamínicos, antibióticos, aminoácidos, etc., a finales de los años sesenta iniciaron el desarrollo de una línea de alimentos infantiles ${ }^{9}$ bajo la denominación Nutribén ${ }^{\circledast}$ y disfrutaban de licencias de los laboratorios Jacquemarie, fundados en 1881 por L. Jacquemaire (1850-1907), elaboradores de productos tan emblemáticos como Bledine o la leche Alma. En 1985, Alter comercializó la leche infantil Natal.

\section{Cheminter S.A.}

Con el inicio de las actividades comerciales de su división dietética en la calle Córcega 541 de Barcelona en la década de los sesenta ${ }^{10,11}$, esta sociedad, inicialmente denominada "Cheminter S.A., Sociedad Internacional Distribuidora Farmacéutica”, modificó su denominación por Cheminter S.A. en 1980 siendo sus objetivos "la distribución y venta de especialidades farmacéuticas, veterinarias, cosméticas, dietéticas y alimentarias y la fabricación de especialidades dietéticas y alimenticias", y se trasladó a la calle Capitán Haya 9 de Madrid. Durante esos años, esta sociedad comercializaba la leche infantil denominada Materlat.

\section{José Sánchez Peñate (JSP)}

Empresa fundada por José Sánchez Peñate (1909-1994) en 1963, en la isla de Gran Canaria. Si bien el objetivo inicial era la comercialización de productos alimenticios, pronto amplió sus actividades con la producción de preparados lác- 
abla Leches infantiles de 1985 y 1990 clasificadas por tipo, indicación en su caso, vigencia durante la década y empresa elaboradora

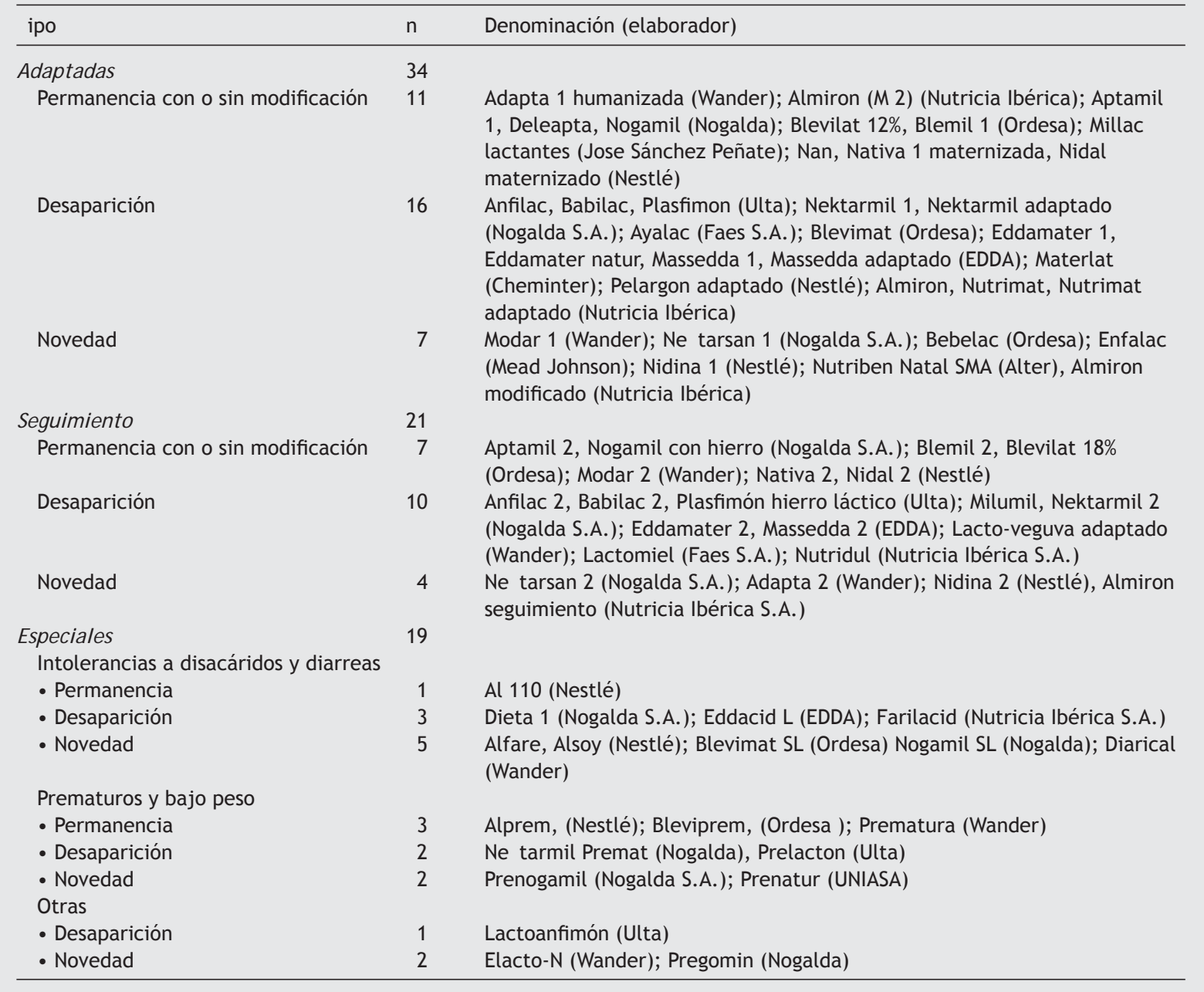

ticos. Durante la década de los ochenta comercializaba la leche Millac lactante, que con modificaciones se continúa elaborando actualmente ${ }^{12}$.

\section{ead Johnson ( ristol ers S.A.E)}

Compañía creada en 1905 por Ed ard Mead Johnson en Nueva Jersey. Interesado por los temas relacionados con la alimentación infantil, introdujo el uso de maltodextrina (Dextri-Maltose ${ }^{\circledR}$ ) en 1911. Bajo la dirección de Daniel Mead Johnson, la empresa se trasladó a Evansville. En 1959 elaboraba las leches Pablum y Enfamil, esta última comercializada en España con la denominación Enfalac desde 1978. Enfalac ha sido objeto de varias formulaciones posteriores. En 1968, Mead Johnson Co. fue adquirida por BristolMyers Co. (empresa creada en 1898 por Bristol y Myers a partir de una compañía farmacéutica), que a su vez, en 1989, se fusionó con E.R. Squibb ${ }^{13}$.

\section{utricia Ibérica S.A.}

Con orígenes en el año 1896, la compañía fue creada en Holanda por los hermanos Van der Hagen en 1901. A mediados de los años setenta la filial española, Nutricia Ibérica S.A., comercializaba las leches Almirón. En 1990, Nutricia adquirió la firma Milupa y más tarde se fusionó con la marca inglesa Cow \& Gate para dar lugar a Numico, que finalmente fue adquirido en 2007 por el grupo Danone.

\section{IASA E A Pule a}

Esta empresa tiene sus orígenes en la Unión Vinícola Industrial (1910), que posteriormente (1954) pasó a denominarse Unión Industrial y Agroganadera S.A. (UNIASA), dedicada al suministro de leche a la ciudad de Granada (Central lechera de Granada). Más tarde (década de los sesenta) diversificó la producción (leche en polvo, mantequilla, batidos, etc.). 
abla Medianas correspondientes a los valores de composición centesimal (\%) y energético (kcal/100) de las leches adaptadas, de seguimiento y especiales comercializadas en España en los años $1985^{16}$ y $1990^{17}$

\begin{tabular}{|c|c|c|c|c|c|c|}
\hline & \multicolumn{2}{|c|}{ Adaptadas } & \multicolumn{2}{|c|}{ Seguimiento } & \multicolumn{2}{|c|}{ Especiales } \\
\hline & $n$ & Media DE & $\mathrm{n}$ & Media DE & $n$ & Media DE \\
\hline \multicolumn{7}{|l|}{1985} \\
\hline Proteína & 23 & $13,3^{\mathrm{a}} \quad 1,4$ & 13 & $15,7^{\mathrm{b}} \quad 2,0$ & 7 & $15,8^{\mathrm{b}} \quad 3,5$ \\
\hline Hidratos de carbono & 23 & $55,5 \quad 3,0$ & 13 & $58,3 \quad 4,1$ & 7 & $55,6 \quad 2,9$ \\
\hline Lípidos & 22 & $25,5^{\mathrm{b}} \quad 4,3$ & 13 & $19,8^{\mathrm{a}} \quad 3,5$ & 7 & $22,8^{\mathrm{ab}} \quad 2,1$ \\
\hline Minerales & 18 & $2,8 \quad 0,71$ & 11 & $3,3 \quad 0,82$ & 6 & $3,5 \quad 1,8$ \\
\hline Energía & 22 & $507^{\mathrm{b}} \quad 25$ & 13 & $471^{\mathrm{a}} \quad 27$ & 7 & $497^{\mathrm{b}} \quad 8,8$ \\
\hline \multicolumn{7}{|l|}{1990} \\
\hline Proteína & 17 & $12,6^{\mathrm{a}} \quad 1,4$ & 11 & $15,9^{\mathrm{b}} \quad 2,3$ & 13 & $14,2^{\mathrm{ab}} \quad 1,6$ \\
\hline Hidratos de carbono & 17 & $55,7 \quad 3,1$ & 11 & $56,5 \quad 2,6$ & 13 & $55,3 \quad 2,9$ \\
\hline Lípidos & 17 & $26,4^{\mathrm{b}} \quad 4,1$ & 11 & $21,2^{\mathrm{a}} \quad 2,6$ & 13 & $24,9^{\text {ab }} \quad 3,3$ \\
\hline Minerales & 13 & $2,5 \quad 0,63$ & 10 & $3,4 \quad 0,65$ & 10 & $3,0 \quad 1,4$ \\
\hline Energía & 16 & $511^{\mathrm{b}} \quad 25$ & 10 & $484^{\mathrm{a}} \quad 15$ & 11 & $502^{\mathrm{ab}} \quad 18$ \\
\hline
\end{tabular}

En 1982, adquirió el 100\% de la Compañía de Dietéticos y Alimentación S.A. (EDDA), empresa que disponía de instalaciones ubicadas en Campello (Alicante), donde elaboraba diferentes preparados infantiles (Eddamater, Massedda, Eddarroz, Eddacereales, etc.), y con ello se propició su entrada en el sector de la alimentación infantil (leche en polvo y harinas (acteadas) ${ }^{14}$.

\section{Productos}

Las leches comercializadas durante el periodo objeto de estudio se relacionan en la tabla 1 , con datos procedentes del Consejo General de Colegios Oficiales de Farmacéuticos ${ }^{16,17}$. Cabe señalar que, del total de fórmulas de la década (n 72),

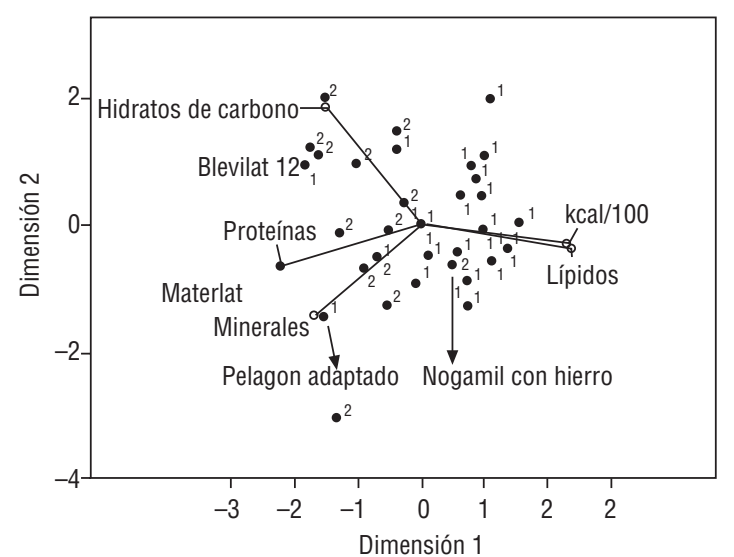

durante este periodo se produjo la modificación de algunas de ellas (un 32\%), la desaparición de otras (un $42 \%$ ) y la introducción de nuevas (un 26\%). A partir de los datos de composición publicados por las empresas, se ha realizado un análisis estadístico de los distintos tipos (adaptadas, seguimiento y especiales) (tabla 2 y fig. 1), así como un estudio cualitativo de ingredientes adicionados (tabla 3 ).

\section{Comentarios}

1. Durante estos años, empresas ya existentes en nuestro país consolidaron su presencia en el sector (Nestlé, Ordesa, Wander) o iniciaron su actividad en él (Alter S.A., Cheminter S.A., JSP, EDDA). Otras, de carácter internacional, ins-

B

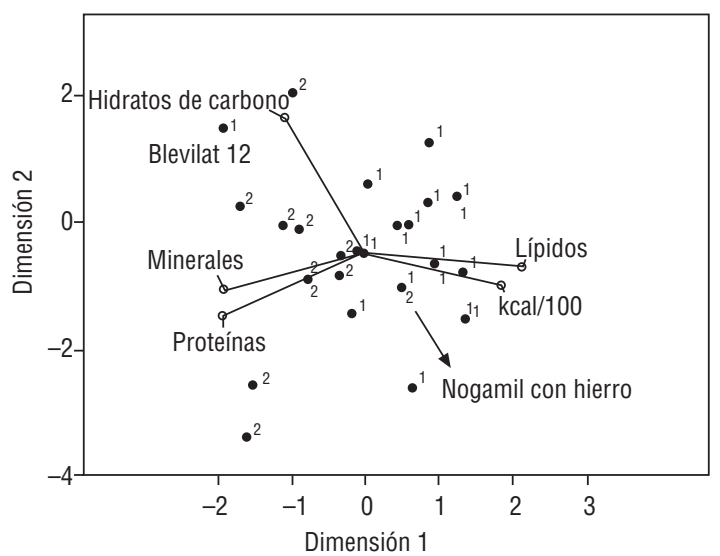

i ura Análisis de componentes principales aplicado a los contenidos en proteína, hidratos de carbono, lípidos y minerales, así como del valor energético, en función del tipo de leche adaptadas (1) y de seguimiento (2) en 1985 (A) y 1990 (B). 
abla Porcentajes de muestras de las leches adaptadas y de seguimiento a las que se adicionaban diferentes ingredientes

\begin{tabular}{lcc}
\hline Ingrediente & Adaptadas, \% & Seguimiento, \% \\
\hline Suero láctico & 100 & 45 \\
$\quad$ desmineralizado & & \\
Aminoácidos & 5 & 0 \\
Sacarosa & 16 & 18 \\
Dextrinomaltosa & 28 & 82 \\
Polisacáridos & 0 & 36 \\
Aceite vegetal & 94 & 100 \\
Grasa animal & 11 & 18 \\
Lecitina & 33 & 27 \\
Vitaminas y minerales & 100 & 100 \\
$\quad$ aurina & 50 & 9 \\
Biotina & 72 & 64 \\
Colina & 50 & 36 \\
Inositol & 50 & 45 \\
\hline
\end{tabular}

talaron filiales en España (Mead Johnson, Nutricia).

2. Se observa que el mayor porcentaje de innovación se produjo en el apartado de las leches especiales (prematuros, intolerancias, alergias) y también que los porcentajes correspondientes a las leches adaptadas y de seguimiento que desaparecieron del mercado fueron relativamente elevados, mientras que la mayoría de las demás sufrieron la modificación de sus fórmulas originales.

3. A partir de los datos recogidos sobre composición de las leches de los años 1985 y 199016,17, se ha realizado un análisis de la varianza para determinar si hay diferencias entre los distintos grupos (adaptadas, seguimiento y especiales) en relación con su contenido en proteínas, hidratos de carbono, lípidos, minerales y energía ( $p 0,05$ ). Se ha utilizado la prueba de Scheffé para separar las medias que mostraran significación $(\alpha \quad 0,05)$. El análisis realizado con los datos correspondientes a las leches de 1985 muestra que no hay diferencias entre grupos en el contenido en hidratos de carbono y minerales, pero sí en los contenidos en proteínas (en las leches adaptadas es inferior que en las leches de seguimiento y especiales) y lípidos (que es mayor en las adaptadas). Este hecho comporta que el aporte energético de las leches adaptadas (y especiales) sea mayor que el de las leches de seguimiento, de modo que cumplen las recomendaciones de la ESPGHAN. Estos resultados son semejantes a los obtenidos con las leches incluidas en el catálogo de $1990^{17}$.

4. En la misma línea del apartado anterior, el análisis de componentes principales realizado muestra que se puede discriminar las leches adaptadas de las de seguimiento (fig. 1) por su contenido lipídico y su aporte energético, mientras que estos mismos parámetros no discriminan las de seguimiento. En relación con las muestras correspondientes a 1990, se observa que, pese a haber disminuido su número, la capacidad de discriminación entre los dos grupos es mayor. Esta resultado indica que se daba un proceso de mejor adecuación de las fórmulas a las recomendaciones existentes y de desaparición de las leches que no las cumplían.

5. El conjunto de las leches adaptadas y de seguimiento muestra la utilización generalizada de aceites vegetales en su formulación, así como el enriquecimiento en vitaminas y sales minerales. En cuanto a la fracción proteica, cabe destacar que todas las leches adaptadas contenían suero láctico desmineralizado (con objeto de mejorar la relación caseína/lactoalbúmina). En cuanto a los hidratos de carbono, el porcentaje de muestras adicionadas de sacarosa era semejante en ambos grupos, mientras que las dextrinomaltosas eran de uso generalizado en las de seguimiento. Por último, cabe destacar que los porcentajes de leches adicionadas de taurina (adaptadas), biotina, colina e inositol eran muy significativos.

\section{Conflicto de intereses}

Ninguno.

\section{iblio ra a}

1. Boatella J. Los primeros preparados destinados a la lactancia materna, registrados en España (1919-1935). Actividad Dietética. 2009;13:173-7.

2. Boatella J, Bou R. Las leches infantiles durante los años cuarenta. Rev Esp Nutr Hum Diet. 2011;15:67-72.

3. Boatella J, Bou R. Las leches infantiles en España durante el periodo 1955-1975: años de transición e innovaciones. Rev Esp Nutr Hum Diet. 2012 [en prensa].

4. ESPGHAN Committee on Nutrition. Guidelines on infant nutrition. I. Recommendations for the composition of an adapted formula. Acta Paediatr Scand. 1977;262 Suppl:1-20.

5. ESPGHAN Committee on nutrition. Guidelines on infant nutrition. II. Recommendations for the composition of follo -up formula and Bei ost. Acta Paediatr Scand. 1981;287 Suppl:1-25.

6. ESPGHAN Committee on Nutrition. Guidelines on infant nutrition. III. Recommendations for infant feeding. Acta Paediatr Scand. 1982;302 Suppl.:1-27.

7. Smith L, Darling P, Roy CC. Pitfalls in the design and manufac ture of infant formulas. En: Lebenthal E, editor. extboo of gastroenterology and nutrition in infancy. 2. ${ }^{\text {a }}$ ed. $\mathrm{Ne}$ or : Raven Press Ltd; 1989.

8. Real Decreto 2685/1976, por el que se aprueba la Reglamentación écnico-Sanitaria para la Elaboración, Circulación y Comercio de Preparados Alimenticios para Regímenes Dietéticos y/o Especiales. BOE 284, de 26/11/1976, p. 23543-9.

9. Disponible en: http://www.laboratoriosalter.es/2/2_1.php

10. La Vanguardia 15/2/1969.

11. La Vanguardia $12 / 5 / 1978$.

12. Disponible en: http://www.jsp.es/jsp/historia.html

13. Disponible en: http://www.meadjohnson.com/Company/Pages/Our-History.aspx

14. Disponible en: http://www.puleva.es/pf/compania/historia. html

15. EDDA. Información técnica. Campello, Alicante: EDDA; 1980.

16. Catálogo de Parafarmacia 85. Madrid: Consejo General de Colegios Oficiales de Farmacéuticos; 1985.

17. Catálogo de Parafarmacia 1990-91. Madrid: Consejo General de Colegios Oficiales de Farmacéuticos; 1991. 